\title{
EOSN - A New TOUGH2 Module for Simulating Transport of Noble Gases in the Subsurface
}

\author{
Chao Shan * and Karsten Pruess \\ Earth Sciences Division, Lawrence Berkeley National Laboratory \\ University of California, Berkeley, CA 94720
}

\begin{abstract}
Noble gases widely exist in nature, and except for radon, they are stable. Modern techniques can detect noble gases to relatively low concentrations and with great precision. These factors suggest that noble gases can be useful tracers for subsurface characterization. Their applications, however, require an appropriate transport model for data analyses. A new fluid property module, EOSN, was developed for TOUGH2 to simulate transport of noble gases in the subsurface. Currently any of five different noble gases (except radon) as well as $\mathrm{CO}_{2}$ can be selected, two at a time. For the two selected gas components, the Crovetto et al. (1982) model is used to calculate the Henry's law coefficients; and the Reid et al. (1987) correlation is used to calculate the gas phase diffusivities. Like most other sister modules, TOUGH2/EOSN can simulate nonisothermal multiphase flow and fully coupled transport in fractured porous media. Potential applications of the new module include, but are not limited to: a) study of different reservoir processes such as recharge, boiling, condensation, and fracture-matrix fluid exchange; b) characterization of reservoir geometry such as fracture spacing; and c) analysis of $\mathrm{CO}_{2}$ sequestration.
\end{abstract}

Key words: TOUGH2 module, noble gas transport, temperature effects.

*Corresponding author. Fax: 510-486-8694; E-mail: c_shan@lbl.gov 


\section{Nomenclature}

$a_{0}, a_{1}, a_{2}, a_{3}$ - experimentally-determined parameters for calculating the Henry's law coefficient, $\mathrm{K}_{\mathrm{h}}$

A, B, C, D, E, F G, H - gas-dependent constants for calculating $\Omega_{\mathrm{D}}$

$\mathrm{d}$ - diffusion coefficient

$\mathrm{D}_{\mathrm{AB}}$ - diffusivity of gas $\mathrm{A}$ in gas $\mathrm{B}$

$\mathrm{f}-$ mass flux

$\mathrm{k}$ - Boltzmann constant

$\mathrm{M}_{\mathrm{AB}}$ - representative molecular weight of the gases A and $\mathrm{B}$ mixture

$\mathrm{K}_{\mathrm{h}}-$ Henry's law coefficient

$\mathrm{P}$ - partial pressure

$\mathrm{T}^{*}$ - modified temperature

$\mathrm{T}$ - temperature in $\mathrm{K}$

$\mathrm{t}$ - one thousandth of $\mathrm{T}$

$x-$ mol fraction

$\mathrm{X}$ - mass fraction

\section{Greek Symbols}

$\beta-$ phase number

$\varepsilon_{\mathrm{AB}}$ - geometric mean characteristic Lennard-Jones energy of gases A and B

$\phi-$ porosity

$\kappa-$ component number

$\rho-$ density 
$\sigma_{\mathrm{AB}}-$ characteristic length

$\tau-$ tortuosity

$\Omega_{D}-$ dimensionless coefficient
Abbreviations
$a q-$ aqueous
NCG - non-condensable gas
TD - temperature dependence 


\section{Introduction}

The natural availability and chemical stability make some gases, especially noble gases good tracers in studying transport processes in the subsurface. Two important mechanisms that affect multi-phase transport are phase partitioning and gas diffusion. For trace gases, phase partitioning can be described using Henry's law, and gas diffusion using Fick's law. Each law is characterized by a coefficient: the Henry's law coefficient and the gas diffusivity. Although both coefficients were assumed constant in many applications, laboratory experiments have shown that they are temperature/pressure dependent. For noble gases and some other gases such as carbon dioxide $\left(\mathrm{CO}_{2}\right)$, this thermodynamic effect is so strong that it may significantly affect results in cases of large temperature variation. In fact, the dependence of noble gas solubility on temperature is so strong as to make monitoring of noble gas concentration in subsurface waters of meteoric origin a useful tool for determining recharge temperature (Ballentine and Hall, 1999). Information on multi-phase processes (boiling, condensation) can also be obtained.

Among many TOUGH2 fluid property modules, EOS7R is the one that can simulate two-phase, five-component flow and transport problems. The two phases are the water and gas phases, and the five components are water, brine $(\mathrm{NaCl})$, air (an mixture of $21 \%$ of oxygen and $79 \%$ of nitrogen), and two radionuclides (Oldenburg and Pruess, 1995). Pruess et al (2000) used TOUGH2 with the EOS7R module to simulate the transport of different tracers in a geothermal reservoir. These tracers included R-134a, tritium, neon, and xenon. The original TOUGH2, however, required users to input Henry's law coefficient and gas diffusivity as constant values. 
A new TOUGH2 module, EOSN is developed to take account of the temperaturedependence of the two coefficients. In applying the new module, users only need to give the names of the two gases; all required thermodynamic properties are provided in an internal data bank. There are, however, options for users to overwrite internal molecular weights for modeling specific isotopes. Currently there are six user-selectable gases: He (helium), $\mathrm{Ne}$ (neon), $\mathrm{Ar}$ (argon), $\mathrm{Kr}$ (krypton), $\mathrm{Xe}$ (xenon), and $\mathrm{CO}_{2}$ (carbon dioxide). $\mathrm{Rn}$ (radon) may be added in the future; a capability to model radioactive decay by means of a half-life is already included in EOSN. The temperature-dependent coefficients are calculated by two new subroutines: NOHEN for calculating the temperature-dependent Henry's law coefficients for the three gas components (air and two user-selected gases), and GASDIF for calculating their temperature and pressure dependent diffusivities in the gas phase. We set diffusivities for the two other components (water and brine) zero in both (aqueous and gas) phases. We also set a default value of $10^{-9} \mathrm{~m}^{2} / \mathrm{s}$ for diffusivities of the three gas components (air and two user-selected gases) in the aqueous phase. There is an option, however, for users to specify aqueous phase diffusivities for all five components through the data block, DIFFU. Theoretical background of the two subroutines is given in the next section. 


\section{Module Description}

TOUGH2 simulates phase partitioning using Henry's law, and gas diffusion using Fick's law. According to Henry's law, the partial pressure of a non-condensable gas $(N C G)$ in the gas phase is proportional to the mol fraction $(x)$ of the dissolved $N C G$ in the aqueous ( $a q)$ phase (Prausnitz et al., 1986):

$$
P_{N C G}=K_{h} x_{a q}^{N C G}
$$

where $K_{h}$ is the Henry's law coefficient.

According to Fick's law, the diffusive flux of component $\kappa$ in phase $\beta$ (= aqueous, gas) is given by (Pruess et al., 1999)

$$
\mathbf{f}_{\beta}^{\kappa}=-\phi \tau_{0} \tau_{\beta} \rho_{\beta} d_{\beta}^{\kappa} \nabla X_{\beta}^{\kappa}
$$

where $\phi$ is porosity; $\tau_{0} \tau_{\beta}$ is the tortuosity that includes a porous medium dependent factor $\tau_{0}$ and a coefficient $\tau_{\beta}$ that depends on phase saturation $S_{\beta}$, i.e. $\tau_{\beta}=\tau_{\beta}\left(S_{\beta}\right) ; \rho_{\beta}$ is density;

$d_{\beta}^{\kappa}$ is the diffusion coefficient of component $\kappa$ in bulk fluid phase $\beta$; and $X_{\beta}^{\kappa}$ is the mass fraction of component $\kappa$ in phase $\beta$. Here, $K_{h}$ and $d_{\beta}^{\kappa}$ are not user-specified constants but code-calculated variables. At every time step and iteration, TOUGH2/EOSN substitutes the most up-to-date temperature/pressure to the two new subroutines (NOHEN and GASDIF) for updating the two coefficients. We use the best available models as the basis of the two subroutines. NOHEN uses the laboratory experiment-based Crovetto et al. (1982) model (using data measured between room temperature and $600 \mathrm{~K}$ ) to calculate the Henry's law coefficient; and GASDIF uses the theoretical model given by Reid et al. 
(1987) to calculate the noble gas diffusivity in the gas phase. Figures 1A and 1B show the temperature-dependent Henry's law coefficients and gas phase diffusivities for air and six gases calculated by the above models. Note that Figure 1B depicts diffusivity at saturation pressure corresponding to temperature. The reason for diffusivities going to such large values at low temperatures is their inverse dependence on pressure.

\section{Henry's Law Coefficients}

NOHEN calculates the Henry's law coefficient $K_{h}$ in (1) using the Crovetto et al. (1982) model:

$$
K_{h}=10^{9} \cdot e^{a_{0}+a_{1} / t+a_{2} / t^{2}+a_{3} / t^{3}}
$$

where the variable, $t$ is one thousandth of temperature in $\mathrm{K}$, i.e.

$$
t=0.001 \times T
$$

Equation (3) is an empirical one that is fitted against the calculated $K_{h}$ using experimental data of Crovetto et al. (1982). The four coefficients in (3) are gas-specific, and their values for seven gases used in TOUGH2 are given in Table 1.

The coefficients for $\mathrm{Ne}, \mathrm{Ar}, \mathrm{Kr}$, and $\mathrm{Xe}$ are directly from Crovetto et al. (1982). The coefficients for He are obtained by fitting the Smith (1985) data for He to the Crovetto et al. (1982) model. For $\mathrm{CO}_{2}$ and air we (Shan and Pruess, 2003) chose four data points for each gas from Naumov et al. (1974), and substituted them into (10). This provided four linear equations for $a_{0}$ through $a_{3}$, which were solved simultaneously to obtain the coefficients for $\mathrm{CO}_{2}$ and air as shown in Table 1. Naumov et al. (1974) only 
provided the Henry's law coefficient data for oxygen and nitrogen (D'Amore and Truesdell, 1988). We assumed that air is simply a mixture of $21 \%$ of oxygen and $79 \%$ of nitrogen, and used the following formula to estimate the Henry's law coefficient for air (Pruess and Battistelli, 2002):

$$
K_{h(A i r)}=1 /\left(0.21 / K_{h\left(O_{2}\right)}+0.79 / K_{h\left(N_{2}\right)}\right)
$$

\section{$\underline{\text { Gas Diffusivity }}$}

GASDIF calculates the diffusivities of three gas components (air and two noble gases) using the following equation modified from Reid et al. (1987):

$$
D_{A B}=0.01881 \times T^{3 / 2} \cdot M_{A B} /\left(P \cdot \sigma_{A B}{ }^{2} \cdot \Omega_{D}\right)
$$

In Equation (6) $D_{A B}$ is the diffusivity of gas A (air or noble gas - component $\kappa$ ) in gas B (water vapor in TOUGH 2 - gas phase), in $\mathrm{m}^{2} / \mathrm{s}$. In fact, $D_{A B}$ represents any specific gas diffusivity of $d_{\beta}^{\kappa}$ in (2). $T$ is temperature, in K. $P$ is pressure, in Pa. $M_{A B}$ is defined by:

$$
M_{A B}=\sqrt{\left(M_{A}+M_{B}\right) /\left(M_{A} \cdot M_{B}\right)}
$$

where $M_{A}$ and $M_{B}$ are the molecular weights of gases A and B, respectively.

The other two parameters in (6) are: $\sigma_{A B}$, a characteristic length, in angstrom; and $\Omega_{D}$, a dimensionless coefficient defined by:

$$
\Omega_{D}=A \cdot\left(T^{*}\right)^{-B}+C \cdot e^{-D T^{*}}+E \cdot e^{-F T^{*}}+G \cdot e^{-H T^{*}}
$$

where $A$ through $H$ are eight gas-independent constants, and $T^{*}$ is a modified temperature that is defined by: 


$$
T^{*}=T /\left(\varepsilon_{A B} / k\right)
$$

where $\varepsilon_{A B}$ is the geometric mean characteristic Lennard-Jones energy of gas $\mathrm{A}$ and $\mathrm{B}$ (Reid et al., 1987); $k$ is the Boltzmann constant. Values of the eight constants are given in Table 2.

The other three parameters, $M_{A B}, \sigma_{A B}$, and $\varepsilon_{A B} / k$ are gas-specific. Their values for five noble gases, carbon dioxide, and air are given in Table 3. Although air is a mixture of different gases (mainly oxygen and nitrogen), here we treat it as a single pseudocomponent.

In TOUGH2 we considered water vapor as Gas B and calculated these gasspecific constants. The rationale is that the thermodynamic effect on gas diffusivity is significant only in an environment with large temperature/pressure variation such as in geothermal reservoirs, where water vapor is the dominant component in the gas phase.

For most other groundwater problems, temperature is in the range of $10{ }^{\circ} \mathrm{C}$ to $20{ }^{\circ} \mathrm{C}$, and air is likely the dominant component in the gas phase. As a result, using the above constants may cause some error. However, the thermodynamic effect is usually very insignificant for those problems and users may not want to consider it at all.

\section{Example Applications}

We use TOUGH2/EOSN to demonstrate temperature effects in two different problems under different conditions. The first problem is the one previously presented by Pruess et al. (2000). The second is a simple one-dimensional advection-diffusion problem with relatively large temperature variations. For both problems, we ran the simulation 
twice: with full temperature dependence of gas diffusivities and Henry's coefficients and without such temperature dependence.

\section{Example 1: Transport of Noble Gases to an Extraction Well}

A central well in a geothermal reservoir with a uniform thickness of 500 meters produces at a constant rate of $20 \mathrm{~kg} / \mathrm{s}$. The reservoir is homogeneous and isotropic such that the flow is radial. An impermeable boundary is set at a radial distance of 1000 meters. The reservoir has the following initial conditions: pressure of 33.479 bar, gas saturation of $20 \%$ (or liquid saturation of $80 \%$ ), temperature of $240{ }^{\circ} \mathrm{C}$, and aqueous

phase mass fractions of $10^{-12}, 2.44 \times 10^{-11}$, and $2.142 \times 10^{-11}$ for brine, neon (Ne), and xenon (Xe), respectively. The mass fractions of the noble gases correspond to equilibrium solubility at $T=10{ }^{\circ} \mathrm{C}$ for atmospheric abundances of isotopes, ${ }^{22} \mathrm{Ne}$ and ${ }^{132} \mathrm{Xe}$. Our interests are the variations of mass fractions (in gas phases) of $\mathrm{Ne}$ and $\mathrm{Xe}$ at the production well.

Using MESHMAKER of TOUGH2 we divided the domain into 31 elements along the radial direction. We then simulated the production process for 30 years. The result shows a clear decreasing trend for pressure, temperature, water saturation, and mass fractions of noble gases towards the production well. The time-varying gas phase mass fractions of noble gases at the production well are plotted in Figure 2. Results with and without temperature dependence (TD) are virtually identical in this problem. 


\section{Example 2: One-Dimensional Noble Gas Transport}

In this hypothetical problem we have a 10-meter long horizontal homogeneous porous rock column with a uniform cross-sectional area of $1 \mathrm{~m}^{2}$. The column has a low permeability of $10^{-18} \mathrm{~m}^{2}$ (1 microdarcy) and is initially at equilibrium condition with a pressure of 24 bar, a gas saturation of $10 \%$, and a temperature of $200{ }^{\circ} \mathrm{C}$. The initial mass fractions for brine and two noble gases ( $\mathrm{Ne}$ and $\mathrm{Xe}$ ) are exactly the same as those in sample problem 1. Mass and heat are removed at the surface of one end of the column (the discharge face) by setting constant pressure of 1 bar and temperature of $50{ }^{\circ} \mathrm{C}$ at the surface, where noble gas concentrations are assumed zero. A no-flow boundary condition is assigned to the other end of soil column. We are interested in the variations of mass fractions of $\mathrm{Ne}$ and $\mathrm{Xe}$ in the column.

Starting from the no-flow end we divided the domain into 10 elements along its length. Each element is one meter long, numbered sequentially from the no-flow end. The result shows a clear decreasing trend for pressure, temperature, and water saturation towards the discharge face. The mass fractions of air and noble gases in the gas phase, however, show a non-monotonic behavior. This occurs because the lowered temperature causes water vapor condensation and thus the mass fractions for non-condensable components increase. The process is simulated for 5 years. The calculated gas-phase mass fractions of $\mathrm{Ne}$ and $\mathrm{Xe}$ at the discharge face are shown in Figure 3. Results with temperature dependence (with TD) and those without temperature dependence (without TD) agree at early time (note that the coordinates are both on logarithm scale) when both diffusion and temperature drop are insignificant, and start departing later when both 
diffusion and temperature drop become significant. The temperature in the column falls to about $50{ }^{\circ} \mathrm{C}$ at the end of simulation, when diffusivities for both neon and xenon are more than 50 times larger than the corresponding diffusivities at $200{ }^{\circ} \mathrm{C}$ (Figure 1B). That explains why the results accounting for temperature effects (the markers) exhibit faster decrease in Figure 3. 


\section{Conclusions}

TOUGH2/EOSN can simulate noble gas transport in the subsurface, which may provide useful information on reservoir processes and conditions. The temperature-effect may play an important role in gas diffusion-dominant processes and in fluid exchange between matrix blocks and surrounding fractures. The difference of solubility and diffusivity between two different noble gases leads to a difference in resulting noble gas mass fractions (or concentrations), which may provide additional information for subsurface studies. Vaporization and condensation of water may greatly affect mass fractions of gases, which is a factor to be considered in data analyses. The study of noble gases may be extended to any other non-condensable gases or even volatile organic chemicals (VOCs).

\section{Acknowledgment}

This work was supported by the Assistant Secretary for Energy Efficiency and Renewable Energy, Office of Geothermal Technologies, of the U.S. Department of Energy under contract No. DE-AC03-76SF00098. We thank G. Moridis, T. Xu, and M. Adams for reviewing the manuscript. 


\section{References}

Ballentine, C. J., Hall, C. M., 1999. Determining paleotemperature and other variables by using an error-weighted, non-linear inversion of noble gas concentrations in water, Geochimica et Cosmochimica Acta, 63(16): 2315-2336.

Crovetto, R., Fernandez-Prini, R., Japas, M. L., 1982. Solubilities of inert gases and methane in $\mathrm{H} 2 \mathrm{O}$ and in $\mathrm{D} 2 \mathrm{O}$ in the temperature range of 300 to $600 \mathrm{~K}, \mathrm{~J}$. Chem. Phys. 76(2): 1077-1086.

D’Amore, F., Truesdell, A. H., 1988. A review of solubilities and equilibrium constants for gaseous species of geothermal interest, Sci. Geol. Bull., 41(3-4): 309-332.

Naumov, G. B., Ryzhenko B. N., Khodakovsky, I. L., 1974. Handbook of Thermodynamic Data, English edition translated from Russian by USGS, Report USGS-WRD-74-001, Distributed by NTIS, U.S. Dept. of Commerce.

Oldenburg, C. M., Pruess, K., 1995. EOS7R: Radionuclide Transport for TOUGH2, Lawrence Berkeley Laboratory Report LBL-34868, Berkeley, CA.

Prausnitz, J. M., Lichtenthaler R. N., de Azevedo, E. G., 1986. Molecular Thermodynamics of Fluid-Phase Equilibria, Prentice-Hall Inc., Englewood Cliffs, NJ.

Pruess, K., Battistelli A., 2002. TMVOC, A Numerical Simulator for Three-Phase NonIsothermal Flows of Multicomponent Hydrocarbon Mixtures in SaturatedUnsaturated Heterogeneous Media, Lawrence Berkeley National Laboratory Report LBNL-49375, Berkeley, CA.

Pruess, K., Oldenburg, C., Moridis, G., 1999. TOUGH2 User's Guide, Version 2.0, Lawrence Berkeley National Laboratory Report LBNL-43134, Berkeley, CA. 
Pruess, K., O’Sullivan, M. J, Kennedy, B. M., 2000. Modeling of phase-partitioning tracers in fractured reservoirs, Proceedings, Twenty-Fifth Workshop on Geothermal Reservoir Engineering, Stanford University, Stanford, California, January 24-26, SGP-TR-165, LBNL-44802.

Reid, R. C., Prausnitz, J. M., Poling, B. E., 1987. The Properties of Gases and Liquids, McGraw-Hill, New York.

Shan, C., Pruess, K., 2003. EOSN: A TOUGH2 Module for Noble Gases, Lawrence Berkeley National Laboratory Report LBNL-52379, Berkeley, CA.

Smith, S. P., 1985. Noble gas solubility in water at high temperature, Trans. Am. Geophys. Un. (EOS), v 66, p. 397. 


\section{Figure Captions:}

Fig. 1A. Henry's law coefficient as a function of temperature for air and six gases

Fig. 1B. Diffusivity of air and six gases in saturated water vapor as a function of temperature

Fig. 2. Produced noble gas mass fractions for Example 1

Fig. 3. Noble gas mass fractions at the discharge face for Example 2 


\section{Table Captions:}

Table 1. Coefficients used to calculate Henry's law coefficients for seven gases

Table 2. Constants for calculating the dimensionless parameter, $\Omega_{\mathrm{D}}$

Table 3. Gas parameters 


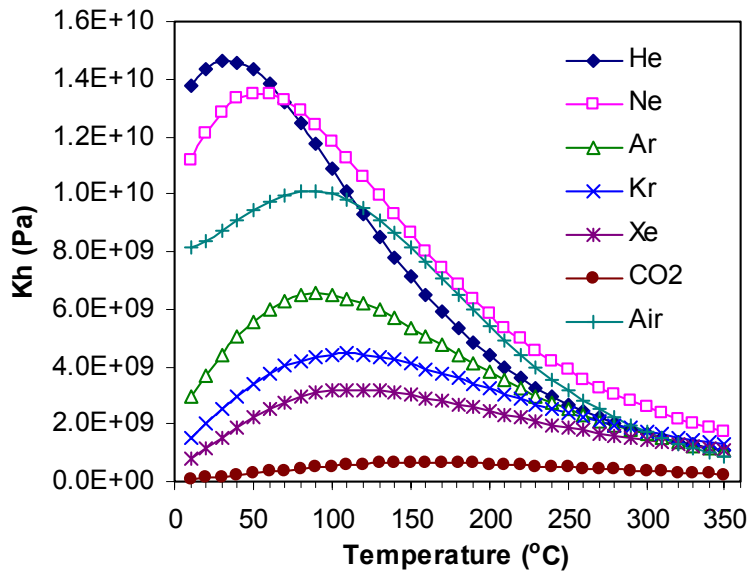




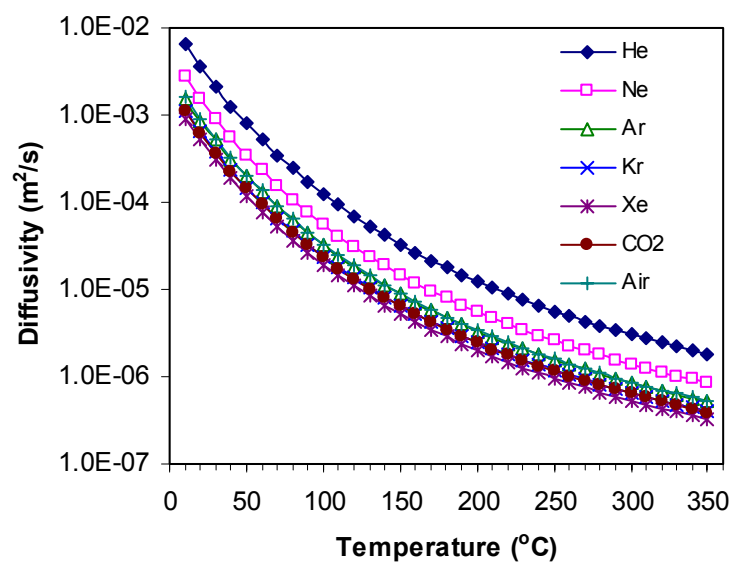




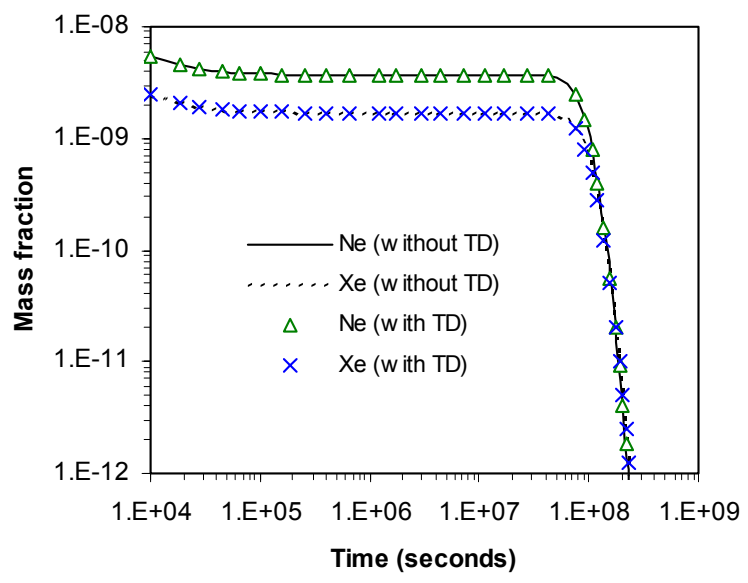




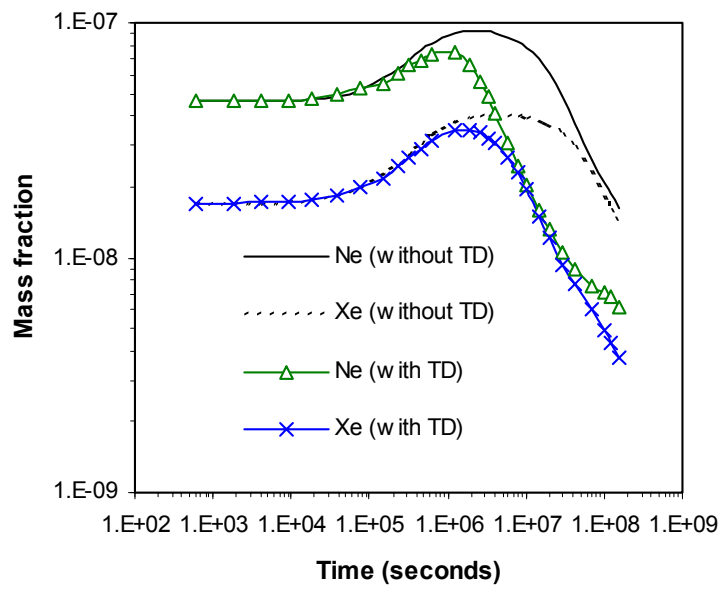




\begin{tabular}{|c|c|c|c|c|}
\hline & $a_{0}$ & $a_{1}$ & $a_{2}$ & $a_{3}$ \\
\hline $\mathrm{He}$ & -8.7928 & 7.8479 & -1.5738 & 0.0755 \\
\hline $\mathrm{Ne}$ & -7.2590 & 6.9500 & -1.3826 & 0.0538 \\
\hline $\mathrm{Ar}$ & -9.5200 & 8.8300 & -1.8959 & 0.0698 \\
\hline $\mathrm{Kr}$ & -6.2920 & 5.6120 & -0.8881 & -0.0458 \\
\hline $\mathrm{Xe}$ & -3.9020 & 2.4390 & 0.3863 & -0.2211 \\
\hline $\mathrm{CO} 2$ & -18.0239 & 18.6643 & -6.1679 & 0.6078 \\
\hline Air & -20.0899 & 21.6153 & -6.8549 & 0.7116 \\
\hline
\end{tabular}




\begin{tabular}{|c|c|c|c|}
\hline$A$ & $B$ & $C$ & $D$ \\
\hline 1.06036 & 0.1561 & 0.193 & 0.47635 \\
\hline$E$ & $F$ & $G$ & $H$ \\
\hline 1.03587 & 1.52996 & 1.764744 & 3.89411 \\
\hline
\end{tabular}




\begin{tabular}{|c|c|c|c|}
\hline & $M_{A B}$ & $\sigma_{A B}$ & $\varepsilon_{A B} / k$ \\
\hline $\mathrm{He}$ & 0.552594 & 2.596 & 90.93405 \\
\hline $\mathrm{Ne}$ & 0.317797 & 2.7305 & 162.9064 \\
\hline $\mathrm{Ar}$ & 0.288662 & 3.0915 & 274.7527 \\
\hline $\mathrm{Kr}$ & 0.259692 & 3.148 & 380.4576 \\
\hline $\mathrm{Xe}$ & 0.251205 & 3.344 & 432.3218 \\
\hline $\mathrm{CO} 2$ & 0.279742 & 3.291 & 397.412 \\
\hline Air & 0.300064 & 3.176 & 252.181 \\
\hline
\end{tabular}

